\title{
Top-Bewertungen dank neuartiger SphereTEC-Füllertechnologie
}

Das neue ceram.X universal eignet sich für direkte und indirekte Restaurationen aller Kavitätenklassen im Front- und Seitenzahnbereich. Das Komposit mit den sphärischen vorpolymerisierten Glasfüllern hat jetzt auch in der Langzeitanwendung überzeugt. 35 Zahnärzte kamen nach einer umfangreichen 6-monatigen Testphase in den eigenen Praxen zu ausgezeichneten Bewertungen hinsichtlich Ästhetik und Handhabung: Das Komposit bietet eine exzellente Adaptation und Model-

lierbar-

keit, deckt mit seinem einfa- chen Farbsystem mit nur 5 Farben das gesamte VITA-Spektrum ab und sorgt mit schneller und einfacher Polierbarkeit für herausragenden Glanz. ceram.x universal ist seit September 2015 in den meisten europäischen Ländern erhältlich.Weitere Informationen zum neuen Füllungsmaterial sind beim DENTSPLY Service Center unter der gebührenfreien Rufnummer 00498000735000 sowie im Internet unter www.dentsply.de/CeramX abrufbar. Videos zur SphereTEC-Füllertechnologie gibt es auch unter www.dentsply-spheretec.com sowie auf Facebook unter www. facebook.de/ceram.xuniversal.

Nach einer Pressemitteilung der

Dentsply Detrey GmbH, Konstanz 